\title{
NOTE ON A THEOREM OF MURRAY
}

\author{
GEORGE W. MACKEY
}

1. Introduction. In a recent paper ${ }^{1}[1]^{2}$ Murray has shown that in any reflexive separable $B$ anach space $\mathfrak{B}$ every closed subspace $\mathfrak{M}$ admits what he calls a quasi-complement, that is, a second closed subspace $\mathfrak{N}$ such that $\mathfrak{M} \cap \mathfrak{N}=0$ and such that $\mathfrak{M}+\mathfrak{N}$, the smallest subspace containing both $\mathfrak{M}$ and $\mathfrak{N}$, is dense in $\mathfrak{B}$. It is the purpose of this note to give a simpler proof of the following somewhat more general theorem.

THEOREM. Let $\mathfrak{B}$ be a separable normed linear space (not necessarily reflexive or even complete) and let $\mathfrak{M}$ be a closed subspace of $\mathfrak{B}$. Then there exists a second closed subspace $\mathfrak{R}$ such that $\mathfrak{M} \cap \mathfrak{N}=0$ and $\mathfrak{M}+\mathfrak{R}$ is dense in $\mathfrak{B}$.

In proving this theorem it is convenient to make use of the notion of closed subspace of a linear system discussed at length in Chapter III of [2]. We repeat the necessary definitions here. A linear system $X_{L}$ is an abstract linear space $X$ together with a linear subspace $L$ of the space $X^{*}$ of all linear ${ }^{3}$ functionals defined on $X$. If $l(x)=0$ for all $l$ in $L$ implies that $x=0$ (that is, if $L$ is total) we say that $X_{L}$ is a regular linear system. If $M$ is a subspace of $X[L]$ we denote by $M^{\prime}$ the set of all $l$ in $L[x$ in $X]$ such that $l(x)=0$ for all $x$ in $X$ [ $[$ in $L]$. It is clear that $M \subseteq N$ implies $N^{\prime} \subseteq M^{\prime}$ and that $M^{\prime \prime} \supseteq M$. Since $M^{\prime \prime \prime}=\left(M^{\prime \prime}\right)^{\prime} \subseteq M^{\prime}$ and since $M^{\prime \prime \prime}=\left(M^{\prime}\right)^{\prime \prime} \supseteq M^{\prime}$ it follows that $M^{\prime}=M^{\prime \prime \prime}$ and hence that $M=M^{\prime \prime}$ if and only if $M$ is of the form $N^{\prime}$. A subspace having either and hence both of these properties is said to be closed. We observe that the operation ' sets up a one-to-one inclusion inverting correspondence between the closed subspaces of $X$ and $L$ respectively.

2. Two lemmas. The proof of the theorem is based essentially on the following lemma.

Lemma 1. Let $X_{L}$ be a regular linear system such that both $X$ and $L$ are $\boldsymbol{\aleph}_{0}$ dimensional, that is, have $\boldsymbol{\aleph}_{0}$ independent generators. Then if $M$

Received by the editors August 20, 1945.

${ }_{1}^{1}$ The author is indebted to Professor Murray for letting him read this paper in manuscript form and for several helpful discussions during the preparation of the present note.

${ }^{2}$ Numbers in brackets refer to the bibliography.

${ }^{3}$ By linear we mean additive and homogeneous. 
is any closed subspace of $X_{L}$ there exists a second closed subspace $N$ of $X_{L}$ such that $M+N=X$ and $M^{\prime}+N^{\prime}=L$.

The proof of Lemma 1 is an easy consequence of a second lemma which is proved in its present form on page 171 of [2] and in other forms elsewhere but which we prove again here for completeness.

Lemma 2. Let $X_{L}$ be as in Lemma 1. Then there exist sequences of elements $x_{1}, x_{2}, \cdots$ and $l_{1}, l_{2}, \cdots$ of $X$ and $L$ respectively such that $x_{1}+x_{2}+\cdots=X, l_{1}+l_{2}+\cdots=L$ and $l_{i}\left(x_{j}\right)=\delta_{i}{ }^{j}$ for $i, j=1,2, \cdots$

Proof. Let $y_{1}, y_{2}, \cdots$ and $m_{1}, m_{2}, \cdots$ generate $X$ and $L$ respectively. We define $x_{1}, x_{2}, \ldots$ and $l_{1}, l_{2}, \ldots$ by induction. Let $l_{1}=m_{1}$ and let $x_{1}=y_{n_{1}} / m_{1}\left(y_{n_{1}}\right)$ where $n_{1}$ is the first integer such that $m_{1}\left(y_{n_{1}}\right) \neq 0$. Suppose that $x_{1}, x_{2}, \cdots, x_{k}$ and $l_{1}, l_{2}, \cdots, l_{k}$ have been defined. If $k$ is odd let $n_{0}$ be the first integer such that $y_{n_{0}} \notin x_{1}+x_{2}+\cdots+x_{k}$ and let $x_{k+1}=y_{n_{0}}-\left(l_{k}\left(y_{n_{0}}\right) x_{k}+\cdots+l_{1}\left(y_{n_{0}}\right) x_{1}\right)$. Then let $\bar{n}$ be the first integer such that $m_{n}\left(x_{k+1}\right) \neq 0$ and let $l_{k+1}=\left(m_{n}-\left(m_{n}\left(x_{k}\right) l_{k}+\cdots+m_{n}\left(x_{1}\right) l_{1}\right)\right) / m_{n}\left(x_{k+1}\right)$. If $k$ is even let $n_{0}$ be the first integer such that $m_{n_{0}} \notin l_{1}+l_{2} \dot{+} \cdots+l_{k}$ and let $l_{k+1}$ $=m_{n_{0}}-\left(m_{n_{0}}\left(x_{k}\right) l_{k}+\cdots+m_{n_{0}}\left(x_{1}\right) l_{1}\right)$. Then let $\bar{n}$ be the first integer such that $l_{k+1}\left(y_{n}\right) \neq 0$ and let $x_{k+1}=\left(y_{n}-\left(l_{k}\left(y_{n}\right) x_{k}+\cdots\right.\right.$ $\left.\left.+l_{1}\left(y_{\tilde{n}}\right) x_{1}\right)\right) / l_{k+1}\left(y_{\tilde{n}}\right)$. It follows at once by induction that $l_{i}\left(x_{j}\right)=\delta_{i}{ }^{j}$ for $i, j=1,2, \cdots$ and it is clear that $X=x_{1}+x_{2}+\cdots$ and $L=l_{1}+l_{2}+\cdots$.

Proof of LEMma 1. For definiteness we shall assume that $M$ and $M^{\prime}$ are infinite-dimensional. The only difference in the contrary case is that certain infinite sequences must be replaced by finite ones. That Lemma 2 is true when $X$ and $L$ are finite-dimensional is obvious. Applying Lemma 2 to $M$ and the linear functionals on $M$ defined by the members of $L$ we may infer the existence of sequences of elements $x_{1}, x_{2}, x_{3}, \cdots$ and $m_{1}, m_{2}, m_{3}, \cdots$ of $M$ and $L$ respectively such that $x_{1}+x_{2}+\cdots=M, M^{\prime}+m_{1}+m_{2}+\cdots=L$ and $m_{i}\left(x_{j}\right)=\delta_{i}{ }^{j}$ for $i, j=1,2, \cdots$. Similarly by applying Lemma 2 to $M^{\prime}$ and the linear functionals on $M^{\prime}$ defined by members of $X$ and remembering that $M^{\prime \prime}=M$ we may infer the existence of sequences of elements $f_{1}, f_{2}, f_{3}, \cdots$ and $z_{1}, z_{2}, z_{3}, \cdots$ of $M^{\prime}$ and $X$ respectively such that $f_{1}+f_{2}+\cdots=M^{\prime}, M+z_{1}+z_{2} \dot{+} \cdots=X$ and $f_{i}\left(z_{j}\right)=\delta_{i}{ }^{j}$ for $i, j=1$, $2, \cdots$. Now for each $i$ and $j=1,2, \cdots$ let

$$
\begin{aligned}
y_{j} & =z_{j}-\left(m_{1}\left(z_{j}\right) x_{1}+m_{2}\left(z_{j}\right) x_{2}+\cdots+m_{j}\left(z_{j}\right) x_{j}\right), \\
l_{i} & =m_{i}-\left(m_{i}\left(z_{1}\right) f_{1}+m_{i}\left(z_{2}\right) f_{2}+\cdots+m_{i}\left(z_{i-1}\right) f_{i-1}\right),
\end{aligned}
$$

where $f_{0}$ and $z_{0}$ are to be taken as zero. Then keeping in mind the fact 
that $f_{i}\left(z_{j}\right)=m_{i}\left(x_{j}\right)=\delta_{i}{ }^{i}$ and $f_{i}\left(x_{j}\right)=0$ for $i, j=1,2, \ldots$ it is easy to verify that $l_{i}\left(y_{j}\right)=0$ and $f_{i}\left(y_{j}\right)=l_{i}\left(x_{j}\right)=\delta_{i}{ }^{i}$. The statement of the lemma now follows at once on setting $N=y_{1}+y_{2}+\ldots$. In fact since $x_{1}+x_{2}+\cdots+y_{1}+y_{2}+\cdots=x_{1}+x_{2}+\cdots+z_{1}+z_{2}+\cdots=M+z_{1}$ $+z_{2}+\cdots=X$ and $l_{1}+l_{2}+\cdots+f_{1}+f_{2}+\cdots=l_{1}+l_{2}+\cdots+m_{1}$ $+m_{2}+\cdots=M^{\prime}+m_{1}+m_{2}+\cdots=L$, it is easy to see that $N^{\prime}=l_{1}$ $+l_{2}+\cdots$ and $N^{\prime \prime}=y_{1}+y_{2}+\cdots$. Thus $N^{\prime \prime}=N$ so that $N$ is closed and $M+N=X$ while $M^{\prime}+N^{\prime}=L$.

3. Proof of the theorem. Since $\mathfrak{B}$ is separable it is clear that $\mathfrak{B} \mid \mathfrak{M}$ is also. ${ }^{4}$ It follows then from Théorème 4 on page 124 of [3] that there exists a countable total set of members of the conjugate of $\mathfrak{B} \mid \mathfrak{M}$ and a countable total set of members of the conjugate of $\mathfrak{B}$. Now every member of the conjugate of $\mathfrak{B} \mid \mathfrak{M}$ has associated with it in an obvious fashion a member of the conjugate of $\mathscr{B}$ which vanishes throughout $\mathfrak{M}$. Thus the first countable total set defines a countable set of elements of the conjugate of $\mathfrak{B}$ the intersections of the null spaces of which is $\mathfrak{M}$. Denote the linear span of these two countable subsets of the conjugate of $\mathfrak{B}$ by $L$. Since $\mathfrak{M}$ and $\mathfrak{B}$ are separable there exists a dense countable set in $\mathbb{B}$ a subset of which is a dense set in $\mathfrak{M}$. Let $X$ be the linear span of this countable set and let $M=\mathfrak{M} \cap X$. It is obvious that the $X, L$ and $M$ so defined satisfy the hypotheses of Lemma 1 and that the closures of $M$ and $X$ are $\mathfrak{M}$ and $\mathscr{B}$ respectively. That $M$ is closed as a subspace of the linear system $X_{L}$ follows from the fact that $\mathfrak{M}$ is an intersection of null spaces of members of $L$. Let $N$ be the closed subspace of $X_{L}$ whose existence is guaranteed by Lemma 1 . We define $\mathfrak{R}$ as the closure (in $\mathfrak{B}$ ) of $N$. Since $\mathfrak{M}+\mathfrak{R} \supseteq M+N=X$ it is clear that $\mathfrak{M}+\mathfrak{N}$ is dense in $\mathfrak{B}$. Suppose that $x \in \mathfrak{M} \cap \mathfrak{l}$. Since $\mathfrak{M}$ is the closure of $M$ every element in $M^{\prime}$ vanishes throughout $\mathfrak{M}$. Similarly every element in $N^{\prime}$ vanishes throughout $\mathfrak{R}$. Thus every element in $M^{\prime}+N^{\prime}=L$ vanishes on $x$. But $L$ is total. Therefore $x=0$. Thus $\mathfrak{M} \cap \mathfrak{N}=0$ and the theorem is proved.

4. Remarks. Murray's paper closes with a proof that in reflexive spaces quasi-complements which are not at the same time complements ${ }^{5}$ are very non-unique in the sense that every such both properly contains and is properly contained in other quasi-complements. This theorem and its proof may be extended to the nonreflexive case (but not the incomplete one) by considering the closed subspaces of the linear systems $\mathfrak{B}_{A}$ and $\mathfrak{A}_{B}$ where $\mathfrak{A}$ is the conjugate of $\mathfrak{B}$ rather than those of the Banach spaces $\mathfrak{A}$ and $\mathfrak{B}$. The needed fact that the linear

- Here $\mathfrak{B} \mid \mathfrak{M}$ denotes the quotient or difference space of $\mathfrak{B} \bmod \mathfrak{M}$.

5 Complements of course are not unique either. 
union of a closed subspace with a finite-dimensional one is again closed follows from Theorem III-1 of [2]. The trouble when $\mathfrak{B}$ is not complete is that $\mathfrak{M}^{\prime}$ and $\mathfrak{N}^{\prime}$ may be complementary even when $\mathfrak{M}$ and $\mathfrak{N}$ are not. That this can indeed happen is shown by the first example at the bottom of page 173 of [2].

Using this same device one may carry over Murray's theory of the connection between quasi-complements and closed projections almost word for word to the nonreflexive and noncomplete case.

Making further use of the methods and theorems of Chapter III of [2] one can show that if $\mathfrak{B}$ is separable and $\mathfrak{M}$ is a closed subspace of $\mathfrak{B}$ such that neither $\mathfrak{M}$ nor $\mathfrak{B} \mid \mathfrak{M}$ is finite-dimensional then the quasicomplement of $\mathfrak{R}$ can always be selected so as not to be a complement and that whenever $\mathfrak{B}$ is complete and $\mathfrak{M}$ and $\mathfrak{R}$ are quasi-complementary and not complementary then $\mathfrak{B}$ has infinitely many linearly independent elements $\bmod \mathfrak{M}+\mathfrak{N}$.

\section{BIBLIOGRAPHY}

1. F. J. Murray, Quasi-complements and closed projections in reflexive Banach spaces, Trans. Amer. Math. Soc. vol. 58 (1945) pp. 77-95.

2. G. W. Mackey, On infinite-dimensional linear spaces, Trans. Amer. Math. Soc. vol. 57 (1945) pp. 155-207.

3. S. Banach, Theorie des operations linéaires, Warsaw, 1932.

HARVARD UNIVERSITY 